\title{
The Application Trends of Rapid Prototyping Manufacturing
}

\author{
Xiao Lin QIU, Yi Ren ZHOU ${ }^{a}$, Zhi Qiang GUO, Jian Hui ZHANG \\ Nanchang Institute of Technology, No.901 Heroe Ave, Qingshanhu, Nanchang, Jiangxi, 330044.China
}

\begin{abstract}
The application of prototyping technology is progressing with each passing day, we studied the characteristics of laser stero lithography (LSL) selective laser sintering (SLS), three-dimensional printing (DP), fused deposition modeling (FDM), computer numerical control (CNC) and other rapid prototyping technologies. After discussed these five rapid prototyping technology materials, we presented the hotspot and direction of rapid prototyping technology and look forward to the development of its technique, the expansion of its field and the progress of its academic ideology.
\end{abstract}

Keywords. rapid prototyping, Three-dimensional model, laser, Crafts

\section{Introduction}

Rapid prototyping technology is the generic terms of group technologywhich produces sample pieces and spare parts in accordance with CAD model, developed in recent years. It is the important part of advanced manufacturing technologya and integrates CAD techniques, CNC technology,laser technology, materials technology and other modern scientific technological achievements. Different with traditional manufacturing methods, rapid prototyping parts based on CAD geometry model, adopt laser beam or other methods to form a solid material accumulation part by the software hierarchical and $\mathrm{CNC}$ forming discrete systems.See Figure 1. Due to its complex three-dimensional manufacturing transformed into a series of superimposed two-dimensional manufacturing, which can generate any complex parts without molds and tools, and greatly enhance theproduction efficiency and manufacturing flexibility.

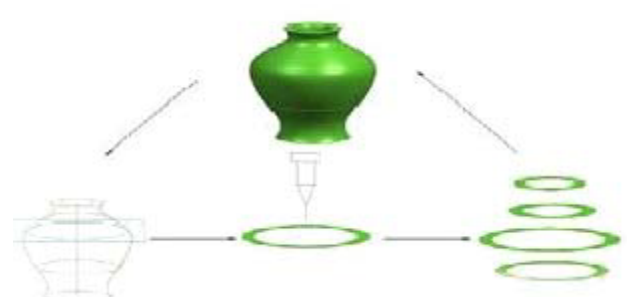

Figure 1 Solid Part generate schematic

(1)The advantages of rapid prototyping technology

Rapid prototyping as a design concept makes an important means of visualization, computer aided design mock-ups of parts can be machined in a very short periodof time, which can quickly design and processing capacity to assess the results. With rapid prototyping and direct digital manufacturing technology that enable cost reduction for the NC 1/3-1/5, cycles $1 / 5-1 / 10$.
(2)Due to the rapid prototyping technology is a complex three-dimensional shape into a two-dimensional crosssection to solve, so it can manufacture high-precision parts arbitrarily complex shaped body, and without any tooling.

(3)Rapid prototyping as an important manufacturing technology, the use of appropriate materials, prototype can be used in subsequent production operations to obtain the final produc in subsequent production operations.

(4)Rapid prototyping can be used in mold manufacturing operation and obtain quickly and economically.

(5)Product manufacturing process is nearly independent of the complexity of the part it can achieve the free form fabrication, which substantially exceeds traditional manuf acturing methods.

\section{The basic principle of rapid prototyping}

Rapid prototyping process which based on the principle of accumulated material actually is manufacturing parts in a discrete layer by layer.To visualize this operation, it could image that the structure of a loaf of bread is a slice of bread fell on top of another piece of bread .There are many rapid prototyping process methods, but all methods are manufacturing layer by layer, the different is the manufacturing methods and materials only.

(1)Geometric Modeling

Get a description of the part CAD files in threedimensional CAD design software (such as ProE \UG SolidWorks $\backslash$ SolidEdge, etc.), as three-dimensional parts 2 (a) in Fig. The general rapid prototyping supported file output format 5TL models that approximating the real surface, the so-called type of surface (Tessellation) treated with flat triangular facets approximation model surface. The advantage of this process is greatly simplified the data format of GAD model, thereby facilitating the subsequent slicing. Because of its relatively simple processing on the data, and regardless of the CAD system, it quickly developed into a standard of rapid prototyping manufacturing data between CAD

\footnotetext{
$\overline{{ }^{a} \text { Corresponding author: renyiz@ } 126 . c o m}$
} 
systems and rapid prototyping machine. Each triangle patch represented with four data items is three vertex coordinates and normal vectors, and the entire CAD model is a collection of such a set of complexors.

When the three-dimensional CAD design software deal with the CAD model surface treatment type, general software system has output accuracy control parameters, by controlling this parameter can be reduced surface approximation error. Such as Pro / E software is selected by chord height value (eh-chord height) as a parameter precision approach, as shown as a sphere, given two value conversions. For a model, give range in software. Under normal circumstances this range can meet project requirements. However, if the value selected is too small, it should abnnegate processing time, storage space, moderately complex parts to be the number of megabytes or even tens of megabytes of storage space. And this data conversion process generates an error can not be avoided, such as the vertices of a triangle in the middle of another side of the triangle, triangle, etc. It will troubled the subsequent data processing, need further examination patch.

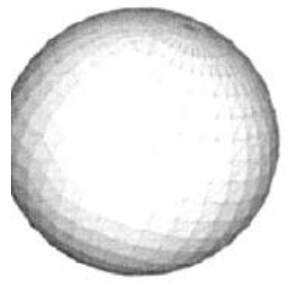

(a)

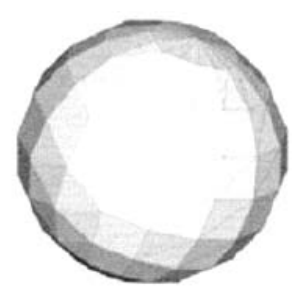

(b)
Figure $2 \mathrm{c} \mathrm{h}$ value of the effectiveness of different (a) $\mathrm{ch}=0.05$ (b) $\mathrm{c} \mathrm{h}=0.2$

(2)Discrete processing three-dimensional model Adopt specified blocking routine to layer a dedicated three-dimensional solid model (typically 5TL model), slicing is after selected production (bulk) direction, should discrete CAD model dimension get every piece of thin cross section contour and entity information. By making a cluster of parallel planes intersects the CAD model, the obtained thin line is the cross sectional contour information, and entity information through somecriterion to obtain. The distance between the parallel planes is layered thickness, i.e. accumulation of the layer thickness during molding. In this process, due to stratification, destroy the continuity of the surface of the slice direction CAD model inevitably lose some of the information model, resulting in part size and shape of errors . Slice thickness layer directly affects the surface roughness of the molding surface of the parts and the entire part of each layer obtained after slicing information is contour information of the upper and lower layers and physical information, and since the contour information is a plane CAD STL file (type of surface after the CAD model) model obtained intersection, so the outline is a series of sequential intersection after intersection even into the fold lin segments, so after stratification model profile has bee obtained by approximation, and contour information has been lost between the layers, thickness ofa large, multi lost information, resulting in an error of the molding surface in the molding process.
Currently, main rapid prototyping process for its classific ation as shown in 3 .

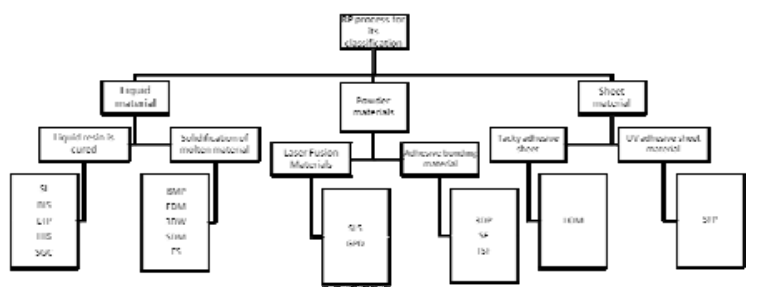

Figure 3 Current major rapid prototyping process for its classification Despite the general steps of various rapid prototyping technologies are the same, but the method for producing aarticle different processes is different, here are several main types of RP processes.

(1) Stereo lithography

Laser stero lithography is a rapid prototyping manufacturing technique most widely used at present.it is actually earlier than fused deposition modeling. It used in the photocurable liquid photosensitive resin is cured (hardened) to a particular shape of the principle. Used thin photosensitive resin as raw materials, according to a predetermined part of each section of the outline layered ultraviolet laser under computer control for track-by-point scanning of the liquid resin, the resin thin layer of the scan area to produce photo-polymerization to form part layer section. Forming at the beginning of the table in its highest position (depth a), at this level is higher than the thickness of a table, part cross-sectional profile of the first layer is scanned, so that the liquid photosensitive resin cured scanning area, forming part of the first a cross-section of the cured layer. Then drop a table thickness, so that the first surface of the cured resin before putting a new layer of liquid resin is then cured repeat the scan, at the same time the new cured layer firmly bonded to the previous layer, the process has been repeat to reach the height $b$. This time has resulted in a fixed wall thickness of the annular cylindrical parts. At this stage it can be noted from the dropped in a vertical direction. After reaching the height $b$, the movement range of the light beam in the $x-y$ plane to produce a flange shape increasing in the front part of the molded part, generally should be added here is similar to FDM support ${ }^{[2]}$. When a certain thickness of the liquid is cured, the process is repeated to produce another cylindrical shaped section from a height of $b$ to $c$. However, around the liquid resin remains flowable, because it is not within the range of the UV beam. Parts thus produced by the lower and upper layers.And when that portion of the liquid resin is not used in the manufacture of other parts can be reused or molding. It may be noted light curing is the same as FDM molding method requires a weak support material, the light curing method.Such a support is used in network structure. Components removed from the end of the table,remove the support structure, you can get three-dimensional parts. 


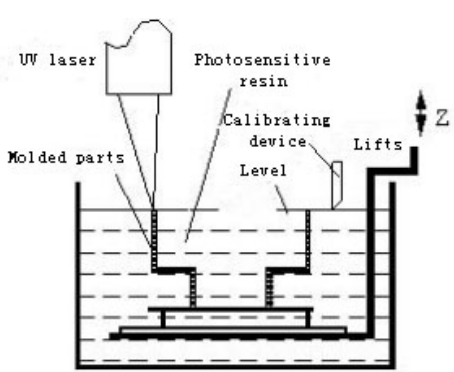

Figure 4 SL artwork

Laser stero lithography can achieve minimum tolerance depends on the extent of the laser focus is usually $0.0125 \mathrm{~mm}$ (O.OOO5in). An inclined surface may also have a good surface quality. RF light curing method is first put into commercial application (Rapid Prototyping) technology. SL (light-curing) equipment accounts for about $70 \%$ RP total number of devices. SL (Stereo Lithography) technology advantages of high accuracy, precision control in general size $0.01 \mathrm{~mm}$; market share, good surface quality, close to $100 \%$ utilization of raw materials, particularly complex shapes can be produced, extremely fine parts and equipment are high. The disadvantage is the need to support the design you can choose the type of material is limited, prone to warpage, materials more expensive. The process is suitable for prototyping and manufacturing complex of small pieces.

(2) selective laser sinering

(Selective Laser Sintering, referred to as SLS) is a non-metallic (metal or Normal) powder to selectively sinter process separate objects. The method uses $\mathrm{CO} 2$ laser as an energy source, currently used in the bottom of the process chamber is equipped with two cylinders:

1) In a powder supply barrel, its internal piston is gradually through a rolling mechanism to enhance the molding parts supply tube powder;

2) The other is part modeling.Its internal piston (table) is gradually reduced to local sintered partially formed.

Firstly, covered with a thin layer $(100 \sim 200 \mu \mathrm{m})$ powder ${ }^{[3]}$ on the table, under computer control the laser beam in accordance with a hierarchical outline part selectively sintered, so that the powder is solidified into a cross-sectional shape, after completion of a drop table layer thickness, the rolling bodies sintered powder spreading surface and then covered with a layer of powder layersintering. Unsintered powder remains loosely retained in theoriginal position, the supporting part being sintered, which secondary deformation limit, without designing a special supporting structure. This process is repeated untilthe entire three-dimensional model manufactured.

After sintering to remove all the excess powder, then grinding and drying process after obtaining the parts needed. Currently, mature technology materials for the wax and plastic powder, direct sintering process of metal powder or ceramic powder is research phase. It is possible to manufacture parts directly to engineering materials, with attractive prospects.

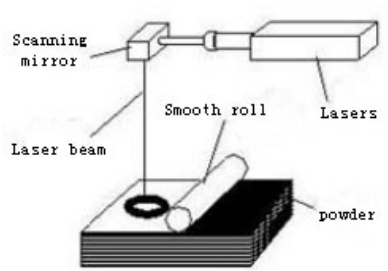

Figure 5 SLS artwork

Advantagesof SLS prototypes are good mechanical properties, high-strength; no need to design and build support; optional material kinds; utilization of raw materials close to $100 \%$, the disadvantages are the prototype of a rough surface; prototypes porous, the need for post processing; high energy consumption; required before processing the material preheating $2 \mathrm{~h}, 5 \sim 1 \mathrm{Oh}$ require cooling after molding, low productivity; molding process requires continuously purged with nitrogen, in order to nsure the safety of the sintering process, high cost; molding process produces toxic gases, certain environmental pollution. SLS process is particularly suitable for the production of functional test parts. Because it can take a variety of different components of the metal powder to sintered copper infiltration performother post-processing, and thus their prototypes may havefabricated metal parts with similar mechanical properties, it can be used directly to manufacture the metal mold. Because the process can be directly sintered wax, in contact with the investment casting process is particularly suitable for small quantities of small complex parts of the production.

(3) 3D Printing

In 1989, Emanuel M. Sachs, John S. Haggerty in Massachusetts Institute of Technology applied for a patent of three-dimensional printing technology, which has become one of the coe patents in this field in the future. Since then, the two researchers repeatedly to modify and improve the technology to get the formation of today's three-dimensional printing Rapid Prototyping (3DP) process. Figure 6 is a simplified schematic of a 3DP process.

3DP technology and selective laser sintering (SLS) have many similarities: both the powder material is selectively bond as a whole. But the biggest difference is that without 3DP the powder material is melted adhesive discharged through the nozzle but it sticking together. Its process usually is: a layer of adhesive is complete, forming cylinder (Fig.14) drops a distance (equal thickness),for the powder cylinder (Fig.18) rise for some height, introduced a number of powder and powder spreading roll onto the forming cylinder, paving and compaction. Head (Fig. 15) under computer control, the construction of a section of press molding data selectivelyspray adhesive construction levels. Dust roller (Fig.13) when the excess powder dusting powder collecting device is collected.

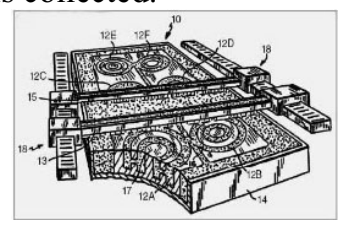

Figure 6 3DP process principle diagram 
Feed powder and dusting and spray adhesives repeated, finalized a three-dimensional bonding powder to produce products.

Dimensional Spray adhesive characteristics: suitable for molding small pieces; the smooth surface of the workpiece is not enough, you need to scan the entire cross-section bonding, forming a long time; the use of multiple nozzles.

(4)Fused Deposition Modeling

As shown in Figure 7, during the process of fused deposition modeling (FDM), the gantry mechanical control head can be moved in two main direction of the table, the tablecan be moved up or down as needed. Thermoplastic or wax extruded from the heating fuse small mouth. The first layer is in accordance with a predetermined trajectory at a fixed rate on the fuse extruded foam matrixformed. Afterthe completion of the first layer, the thickness of a decline bench and began manufacturing superimposed layer. The key is to keep half FDM processflow just above the melting point of the molding material is usually higher than the melting point control in about $1{ }^{\circ} \mathrm{C}$.

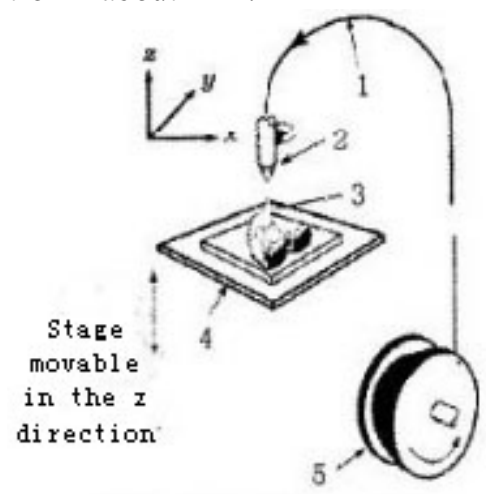

Figure 7 FDM Technology

1, Thermoplastic or wax fuse; 2 , In the $x-y$ plane can move FDM head; 3, Plastic models ; 4, Not fixed base; 5, Provide a fuse

When FDM creating complex parts it must added supporttchnic. Such as the height of the figure7 (a), the next layer of fuse material will be spread without support space. The solution is making a single material support material out of the model, the support material may use low densityfuse, lower than strength material, after the completion of processing of parts it can be removed.

The diameter of the fuse depended on the thickness of themiddle of FDM machine.Usually from $0.5 \mathrm{~mm}$ to $0.25 \mathrm{~mm}(0.02 \mathrm{in}$ to from $0.01 \mathrm{in})$ This value represents the best of tolerance in the vertical direction can be achieved. In the $\mathrm{x}-\mathrm{y}$ plane, as long as the characteristics of the fuse can be extruded, size precision can reach $0.025 \mathrm{~mm}$ (0.001in).

The advantages of FDM is the high utilization, low-cost materials, the choice of the type of multimaterial,processclean, simple, easy to operate and less impact on the environment. The disadvantage is low precision, easy to manufacture complex parts, poor surface quality,low molding efficiency, not suitable for the manufacture of large parts. The process is suitable for conceptual modeling products as well as its shape and function testing, moderately complex forming small, since methacrylic ABS material has good chemical stable, gamma sterilization may be particularly suitable for medical.

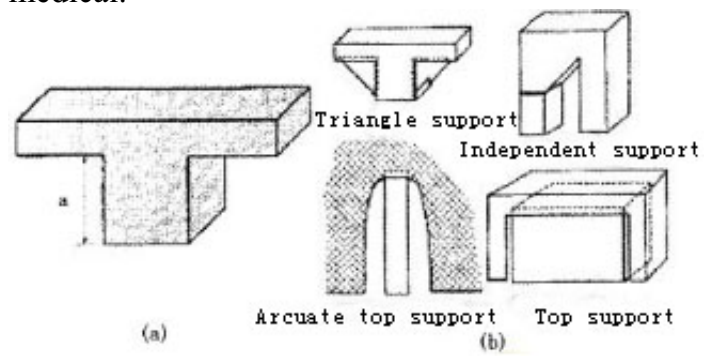

Figure 8 Rapid prototyping support structure diagram

(a)There is a need to highlight the cross-section of the support material parts;

(b)In rapid prototyping machines commonly used in the support structure

(5)Computer Numerical Control

$\mathrm{CNC}$ is an integrated computer-aided design (CAD), computer aided manufacturing (CAM),computer numeric acontrol (CNC) and other advanced technology, the three-dimensional data model on a computer constituted by a single piece of material excavated from cutting. After clamping the workpiece by a digital control system can control the machine according to different processes, and automatically selects the replacement tool automatically change the spindle speed, feed rate and the trajectory of the tool relative to the workpiece and other auxiliary functions, in order to complete the work of several surface working on a multi-step, the whole process is automatically controlled by the program, independent of the operator of human factorsl.

CNC machining method different from the above four RPprocess method, it is "subtractive" digital processing ${ }^{[6]}$, is a modern digital manufacturing plant widely used way of machining, rapid prototyping service providers tend to $\mathrm{CNC}$ machining as a rapid prototyping model supplementary processing, CNC machining high speed, low cost, high precision, large processing size, features a wide range of material selection, to ensure the consistency of the material and surface quality and mechanical properties of production parts and materials may be hand model It reached a high level.

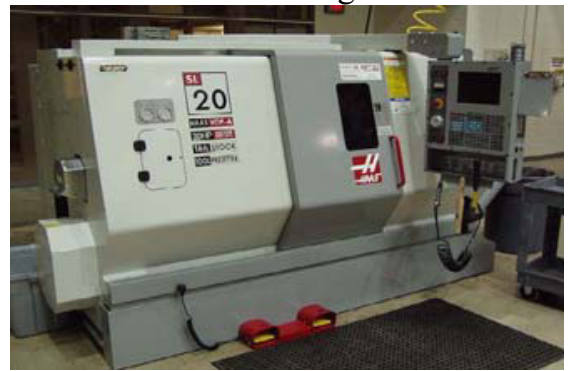

Figure 9 CNC machine tools

(6)Mainstream rapid prototyping process (including CNC) Comparison

SL, SLS, 3DP, FDM, CNC rapid prototyping several respective characteristics are shown in Table 1. 
Table 1 Comparison of mainstream rapid prototyping process

\begin{tabular}{|c|c|c|c|c|c|}
\hline & SL & SLS & 3DP & FDM & $\mathrm{CNC}$ \\
\hline $\begin{array}{c}\text { Forming } \\
\text { speed }\end{array}$ & Faster & Slow & Slow & Slow & Fast \\
\hline $\begin{array}{l}\text { Forming } \\
\text { accuracy }\end{array}$ & Higher & Lower & Lower & Lower & Higher \\
\hline $\begin{array}{c}\text { manufacturing } \\
\text { cost }\end{array}$ & Higher & Lower & low & Lower & Lower \\
\hline Complexity & medium & complex & medium & medium & $\begin{array}{l}\text { Complex } \\
\quad \text { (and } \\
\text { associated } \\
\text { multi-axis) }\end{array}$ \\
\hline Parts size & $\begin{array}{l}\text { Small } \\
\text { pieces }\end{array}$ & $\begin{array}{l}\text { Small } \\
\text { pieces }\end{array}$ & $\begin{array}{l}\text { Small } \\
\text { pieces }\end{array}$ & $\begin{array}{l}\text { Small } \\
\text { pieces }\end{array}$ & The large \\
\hline $\begin{array}{c}\text { Commonly } \\
\text { used materials }\end{array}$ & $\begin{array}{l}\text { Thermoset } \\
\text { ting } \\
\text { photosensi } \\
\text { tive resin }\end{array}$ & $\begin{array}{l}\text { Paraffin } \\
\text { wax, } \\
\text { plastic, } \\
\text { metal, } \\
\text { ceramic } \\
\text { powder, } \\
\text { etc. }\end{array}$ & $\begin{array}{c}\text { ABS } \\
\text { plastic, } \\
\text { which is } \\
\text { also } \\
\text { a mixture } \\
\text { of } \\
\text { aluminum } \\
\text { and glass }\end{array}$ & $\begin{array}{c}\text { Paraffin, } \\
\text { nylon, } \\
\text { ABS, } \\
\text { and } \\
\text { other } \\
\text { low } \\
\text { melting } \\
\text { point } \\
\text { metal }\end{array}$ & $\begin{array}{l}\text { ABS, POM } \\
\text { (race steel), } \\
\text { acrylic, ylon, } \\
\text { PC, } \\
\text { electric } \\
\text { wood. Metal } \\
\text { materials: } \\
\text { aluminum, } \\
\text { magnesium } \\
\text { alloy, zinc } \\
\text { alloy, } \\
\text { copper, etc. }\end{array}$ \\
\hline
\end{tabular}

\section{The direction of rapid prototyping technology development}

Rapid prototyping manufacturing is a method based on the principle of accumulate material, it can be made arbitrarily complex three-dimensional structures. Currently the biggest problem of rapid prototyping development is the technical realization restricted by physical and chemical properties of the material. For example, in the molding material, the key is organic polymer materials; the directly formed of metal material is thehotspot of nearly 10 years research,the difficulty liesin how to improve the accuracy. On the molding material,thedirection of development is the study of ceramic materials and rapid prototyping composites. In the development of manufacturing equipment, 3D printer is hot at home andabroad in recent years, which as a peripheral application of computer. Rapid prototyping technology has a wide application prospect and great commercial value in industrial modeling, product innovation, arts and crafts and so on.

Rapid prototyping technology research focus and direction includes three aspects:

(1) The development of rapid prototyping technology itself. Such as 3D printing make the rapid prototyping into the information market; direct metal forming technology enables the structure and function parts can beproduced directly. Further development is rapid prototyping rapid prototyping technology of ceramic parts and composite materials.

(2) The expansion of rapid prototyping application fields.Such as rapid prototyping applications provide a quick technical support to the automotive manufacture field. Rapid prototyping applications in bioprosthetic tissue engineering, artificial prosthesis manufacture custom providea technical support to the prosthetic manufacture and dimensional scaffold. It is to further the creative design, aerospace manufacturers and the functional structure of devices in the field of development.
(3)The academic thought of rapid prototyping development.Rapid prototyping developed from the shape manufacture in the past to the material organizationand structure design. Try to achieve the organization from micro to the macro-controlled. For example, in the manufacture of composite materials, the ability to accomplish the design of composite material and the manufacture of structure shape. It can achieve the integrati of "design - Material - made" in broader terms.

\section{References}

[1] Hou Shu guang, ZHang Dong, HUANG Xiaoming, ZHAO Yongli. J Investigation of Micro-mechanical Response of Asphalt Mixtures by a Three-dimensional Discrete Element Model. Journal of Wuhan University of Technology(Materials Science Edition).2015 02

[2] Haiyan Liu,Xiaomei Bai,Dan Wei,Gengliang Yang. High-performance liquid chromatography separation of small molecules on a porous poly (trimethylol propane triacrylate-co-N-isopropylacrylamide-co-ethylene dimethacrylate) monolithic column [J]. Journal of Chromatography A2014

[3] Xu Zhifeng, Liang Pei, Yang Wei, Li Sisi, Cai Changchun.Effects of laser energy density on forming accuracy and tensile strength of selective laser sintering resin coated sands. China Foundry. 201403

[4] Yang Ju, Heping Xie, Zemin Zheng, Jinbo Lu, Lingtao Mao, Feng Gao, Ruidong Peng. Visualization of the complex structure and stress field inside rock by means of 3D printing technology. Chinese Science Bulletin. 201436

[5] S.Hwang, E.I.Reyes, N.S.Kim, K.S.Moon, R.C.Rumpf. Parameter study of Fused Deposition Modeling process on thermo-mechanical properties of the final 3D structures made by metal/polymer composite filaments. ICBAEE 2014. BEIJING, CHINA. 2014-05-22. [6] Wang Linyun, Nuang Honghui, Rae W.West, Wang Dzhong. Intelligent manufacturing system of impeller for computer numerical control (CNC) programming based on KBE. Journal of Central South University. 201412 